Rapid Reviews COVID-19

\title{
Reviews of "Development of an automated chemiluminescence assay system for quantitative measurement of multiple anti-SARS-CoV-2 antibodies"
}

Kiang-Teck Yeo ${ }^{1}$, Clarence W Chan ${ }^{2}$, Massimo Pieri ${ }^{3}$

${ }^{1}$ Professor and Director, University of Chicago, Pathology, USA, ${ }^{2}$ Northwestern University, ${ }^{3}$ University of Rome Tor Vergata: Universita degli Studi di Roma Tor Vergata, Experimental Medicine, Italy

Published on: Dec 09, 2020

DOI: $10.1162 / 2 e 3983 f 5 . f 143 f 44 c$

License: Creative Commons Attribution 4.0 International License (CC-BY 4.0). 
To read the original manuscript, click the link above.

Summary of Reviews: The authors develop a new chemiluminescence assay system for measurement of SARS-CoV-2 antibodies. However, reviewers suggest that the study is potentially informative, though the lack of analytical validation in the study limits the generalizability of the findings.

Reviewer 1 (Kiang-Teck Yeo, Clarence W Chan)

Reviewer 2 (Massimo Pieri) |

\section{RR:C19 Strength of Evidence Scale Key}

प्रमप = Misleading

प्र००० = Not Informative

प्रा पि = Potentially Informative

प्र०प्र = Reliable

प्साप्र = Strong

To read the reviews, click the links below. 